\title{
Nkx2-5 mutation causes anatomic hypoplasia of the cardiac conduction system
}

\author{
Patrick Y. Jay, ${ }^{1,2}$ Brett S. Harris, ${ }^{3}$ Colin T. Maguire, ${ }^{1}$ Antje Buerger, ${ }^{2}$ Hiroko Wakimoto, ${ }^{1}$ \\ Makoto Tanaka, ${ }^{2}$ Sabina Kupershmidt, ${ }^{4}$ Dan M. Roden, ${ }^{5}$ Thomas M. Schultheiss, ${ }^{6}$ \\ Terrence X. O’Brien, ${ }^{3,7}$ Robert G. Gourdie, ${ }^{3}$ Charles I. Berul, ${ }^{1}$ and Seigo Izumo ${ }^{2}$
}

\begin{abstract}
1Department of Cardiology, Children's Hospital, Boston, Massachusetts, USA. ${ }^{2}$ Cardiology Division, Beth Israel Deaconess Medical Center, Boston, Massachusetts, USA. ${ }^{3}$ Cardiovascular Developmental Biology Center, Department of Anatomy and Cell Biology, Medical University of South Carolina, Charleston, South Carolina, USA. ${ }^{4}$ Department of Anesthesiology and ${ }^{5}$ Department of Medicine, Vanderbilt University School of Medicine, Nashville, Tennessee, USA. ${ }^{6}$ Division of Molecular Medicine, Beth Israel Deaconess Medical Center, Boston, Massachusetts, USA.

${ }^{7}$ Veterans Administration Medical Center, Charleston, South Carolina, USA.
\end{abstract}

\begin{abstract}
Heterozygous mutations of the cardiac transcription factor $N k x 2-5$ cause atrioventricular conduction defects in humans by unknown mechanisms. We show in KO mice that the number of cells in the cardiac conduction system is directly related to $N k \times 2-5$ gene dosage. Null mutant embryos appear to lack the primordium of the atrioventricular node. In $N k x 2-5$ haploinsufficiency, the conduction system has half the normal number of cells. In addition, an entire population of connexin $40^{-} /$connexin $45^{+}$cells is missing in the atrioventricular node of $N k x 2-5$ heterozygous $\mathrm{KO}$ mice. Specific functional defects associated with $N k x 2-5$ loss of function can be attributed to hypoplastic development of the relevant structures in the conduction system. Surprisingly, the cellular expression of connexin 40, the major gap junction isoform of Purkinje fibers and a putative Nkx2-5 target, is unaffected, consistent with normal conduction times through the His-Purkinje system measured in vivo. Postnatal conduction defects in $N k \times 2-5$ mutation may result at least in part from a defect in the genetic program that governs the recruitment or retention of embryonic cardiac myocytes in the conduction system.
\end{abstract}

\section{Introduction}

During cardiac development, pluripotent myocytes differentiate into specific anatomic and cellular phenotypes. For example, the atrial and ventricular lineages diversify in the precardiac mesoderm even before the heart tube has formed (1). Lineage-tracing analyses in chick embryos likewise show that the conduction system develops by recruitment of adjacent multipotent cardiac myocytes. Recruitment occurs within specific embryonic stages, starting in the central conduction system and ending with the peripheral network of Purkinje fibers $(2,3)$. The cells withdraw from proliferation once they join the system (3). Cell birth dating experiments suggest that the process is the same in the mouse (4). Endothelin and neuregulin-1 in the chick and mouse, respectively, may induce recruitment into the peripheral Purkinje system (5, 6), but little else is known about the mechanisms that govern the development of the conduction system.

To our knowledge, no diseases have yet been reported to perturb specifically the molecular mechanism of the embryonic formation of the conduction system. Physiologic conduction defects commonly result from drugs or mutations that affect the function of ion channels, pumps, or gap junctions, but congenital and acquired anatomic abnormalities do exist. For example, maternal lupus Ab's that cross the placenta destroy cells in the central conduction system of the fetus, causing congenital heart block. The block persists even after the Ab's are no longer present in the infant, consistent with a permanent anatomic lesion (7).

Nonstandard abbreviations used: AV nodal effective refractory period (AVERP); connexin40 (Cx40); embryonic day (E); interventricular septum (IVS); intracardiac electrogram (IEGM); stimulus (S).

Conflict of interest: The authors have declared that no conflict of interest exists.

Citation for this article: J. Clin. Invest. 113:1130-1137 (2004).

doi:10.1172/JCI200419846.
Nkx2-5 is a transcription factor critical for cardiac development from Drosophila to humans (8). Heterozygous mutations of Nkx2-5 in humans cause congenital heart disease. Mutations that affect DNA binding are associated with cardiac conduction defects or heart block $(9,10)$. Nkx2-5 mRNA and protein is transiently upregulated during the formation of conduction fibers relative to the surrounding myocardium in embryonic chick, mouse, and human hearts, suggesting a role in the development of the conduction system (11). We therefore hypothesized that loss of Nkx2-5 function causes hypoplastic development of the conduction system, which in turn contributes to functional defects in the postnatal heart.

\section{Methods}

Generation of mutant strains. The $N k \times 2-5^{+/ n e o}$ line was generated by targeted replacement of the WT allele with the $N k \times 2-5^{\text {neo }}$ construct in AK7 ES cells and injection of positive clones into C57Bl/ 6 blastocysts, as previously described (12). To make the targeting construct, a neomycin resistance cassette flanked by loxP sequences was cloned into the first intron. An additional loxP sequence was cloned after the second exon. ES cells carrying the targeting construct were identified by Southern blot analysis using a $5^{\prime}$ flanking probe. Mice were genotyped by Southern blot analysis or PCR. The minK-lacZ and $N k \times 2-5^{+/-}$lines have been previously described; each was generated by targeted replacement of the minK and $N k \times 2-5$ loci with $\beta$-gal (lacZ) $(12,13)$. Animal care and experiments were done in compliance with Institutional Animal Care and Use Committee guidelines and with the institutional approvals of Children's Hospital and Beth Israel Deaconess Medical Center.

Total RNA was purified from the ventricular myocardium of WT and Nkx2-5 $5^{+/ n e o}$ mice for Northern blot analysis of Nkx2-5 and GAPDH mRNA expression by TRIzol extraction. Total RNA $(10 \mu \mathrm{g})$ was electrophoresed on an agarose gel and transferred 
onto a nylon membrane (Hybond-N; Amersham Biosciences, Piscataway, New Jersey, USA). The membrane was then hybridized, washed, and imaged by standard methods using ${ }^{32}$ P-labeled probes for $\mathrm{Nkx} 2-5$ or GAPDH.

$\beta$-Gal staining and assay. Hearts that expressed minK-lacZ were stained with X-gal for examination of the conduction system as previously described (13). Microscopic sections were obtained from stained hearts embedded in paraffin and eosin counterstained. Hearts viewed in whole mount were dehydrated in a graded methanol series and cleared in benzyl alcohol-benzyl benzoate (1:1). All embryos and mice were genotyped for Nkx2-5 and minKlac $Z$ gene dosage; all comparisons using the minK-lacZ marker were made between hearts bearing equal copy numbers of the minK-lacZ allele, that is, heterozygotes or homozygotes.

Total minK-lacZ enzymatic activity in fetal hearts was quantified by a colorimetric assay. Hearts were dissected from embryonic day 14.5 (E14.5) embryos, washed in PBS, and homogenized in reporter lysis buffer (Promega Corp., Madison, Wisconsin, USA). $\beta$-Gal substrate ( $\mathrm{pH} 7.3,200 \mathrm{mM} \mathrm{NaPO}_{4}, 2 \mathrm{mM} \mathrm{MgCl} 2,100 \mathrm{mM} \beta$-mercaptoethanol, $1.33 \mathrm{mg} / \mathrm{ml}$ o-nitrophenyl- $\beta$-D-galactopyranoside) was mixed with an equal volume of the homogenate and incubated at $37^{\circ} \mathrm{C}$ for 30 minutes. The reactions were stopped with $1 \mathrm{M}$ $\mathrm{Na}_{2} \mathrm{CO}_{3}$. The absorption of the reaction was measured at $420 \mathrm{~nm}$. Measurements were normalized to protein concentration determined by Bradford assays.

Connexin immunohistochemistry. Hearts were snap-frozen in liquid nitrogen, embedded in OCT, and sectioned ( $8-\mu \mathrm{m}$ thickness). Alternate consecutive sections were histochemically stained with H\&E. Immunofluorescent labeling followed previously described methods $(2,3)$. In brief, sections were incubated in primary Ab's overnight at $4{ }^{\circ} \mathrm{C}$. The primary Ab's used were guinea pig anti-rat connexin40 (Cx40), rabbit anti-chick Cx40 (5), rabbit anti-Cx43 (Sigma-Aldrich, St. Louis, Missouri, USA), and guinea pig antiCx45 (14). Secondary Ab's used were goat anti-rabbit Alexa 488 (Molecular Probes Inc., Eugene, Oregon, USA), goat anti-guinea pig TRITC, and goat anti-guinea pig cy5 (Jackson ImmunoResearch Laboratories Inc., West Grove, Pennsylvania, USA). Sections were counterstained with wheat germ agglutinin-TRITC to label cell membranes and Hoechst 33258 to label nuclei (SigmaAldrich). Representative laser-scanning confocal microscopy images at similar short-axis levels of the heart were obtained without knowledge of the Nkx2-5 genotype. Cx40 particle number and size were calculated from ten sections for each heart. Quantitative image analysis was performed using NIH Image (Research Services Branch, National Institutes of Mental Health, Bethesda, Maryland, USA) as described previously (15).

Surface and intracardiac electrophysiology. In vivo cardiac electrophysiologic recordings were obtained in mice at 3 weeks, 7 weeks, and 1 year of age. Three-week-old mice were the youngest age at which their size permitted insertion of the intracardiac catheter. The mice were anesthetized with intraperitoneal pentobarbital $(0.033 \mathrm{mg} / \mathrm{g})$. A surface multilead ECG was obtained through subcutaneous limb leads. ECG signals were sampled at 2,000 Hz, yielding $1 \mathrm{~ms}$ time resolution.

Intracardiac electrogram (IEGM) were obtained by passing a 0.54-mm diameter (1.7 French) octapolar electrode catheter (CIBer mouse EP; NuMed Inc., Hopkinton, New York, USA) into the right heart via the internal jugular vein. The eight electrodes spaced 0.5 $\mathrm{mm}$ apart directly contact the endocardial surface of the heart. Bipolar recordings of the atrial and ventricular depolarization were obtained from adjacent electrodes in the superior right atrium just past the superior vena cava and right ventricular apex, respectively. Recordings of the His bundle were obtained by manipulations of the catheter to obtain a triphasic signal from the middle electrodes. The His amplitude was measured from the maximal signal obtained from the bipolar IEGM from the middle electrodes. Proper catheter placement was confirmed by visual inspection through a sternotomy at the end of studies.

The cardiac intervals, RR, PR, QRS, and QT, were measured from the surface ECG. The intracardiac intervals, $\mathrm{AV}, \mathrm{AH}$, and $\mathrm{HV}$, were measured from the onset of deflection of the atrial, His, and right-ventricular IEGM signals. During normal sinus rhythm, the "A" recording measured from the superior right atrium near the sinus node should be coincident with the onset of the PR interval. In practice, the A measured anywhere in the right atrium of the mouse is nearly simultaneous with the onset of the $\mathrm{P}$ wave on the surface ECG.

Standard clinical electrophysiological pacing protocols were used to determine all basic electrophysiological parameters (16-18). The AV-His-Purkinje conduction properties were assessed by rapid atrial pacing at fixed rates; each subsequent train of stimuli was delivered at 5-ms shorter decrements. The minimum paced cycle length that maintained $1: 1 \mathrm{AV}$ conduction and the maximum cycle length that caused 2:1 AV block were determined for each animal. The 1:1 cycle length is the minimum coupling interval in which $A V$ conduction is maintained with a constant $A V$ interval. The 2:1 cycle length is the maximal interval that causes every other ventricular beat to be refractory to the paced atrial beat. The AV nodal effective refractory period (AVERP) was determined by an S1S2 protocol in which an eight-beat train of stimuli (S1) at 150-ms intervals was followed by a stimulus (S2) at a progressively shorter coupling interval; AVERP was defined as the maximal S1S2 coupling interval that failed to propagate to the ventricle. All recordings and measurements were made without knowledge of genotype.

Statistical analysis. Numerical data are presented as mean plus or minus SEM. Statistical significance for differences between groups was set at $P$ values less than 0.05 as determined by the Student's two-sided $t$ test.

\section{Results}

To examine the anatomy of the embryonic and postnatal conduction system, we used two independent sets of molecular markers. First, we crossed Nkx2-5 haploinsufficient mice (Nkx2$5^{+/ n e o}$ ) with $\operatorname{minK}$-KO mice in which the minK-coding region was replaced with $\beta$-gal (minK-lacZ). MinK-lacZ labels the nuclei of conduction system myocytes, as determined by their anatomic location and coexpression with the gap junction protein connexin $40(13,19)$. The $N k \times 2-5^{\text {neo }}$ allele, originally created for Crerecombinase-mediated conditional deletion experiments, retains a neomycin-resistance cassette and flanking loxP sites in the noncoding regions. The allele unintentionally caused loss of function, presumably because of the neomycin-resistance cassette, as previously reported for other genes $(20,21)$. Nkx2-5 $5^{+/ n e o}$ hearts have half as much Nkx2-5 mRNA as WT (Figure 1A). $N k \times 2-5^{\text {neo/neo }}$ embryos were morphologically identical to previously described homozygous $N k \times 2-5 \mathrm{KO}\left(N k \times 2-5^{-1-}\right)$ embryos (12). The hearts of $N k \times 2-5^{\text {neo/neo }}$ embryos at E9.5 had arrest of cardiac development after partial looping, a bulbous atrium and ventricle, a wide $A V$ canal, and a stenotic outflow tract. No viable 

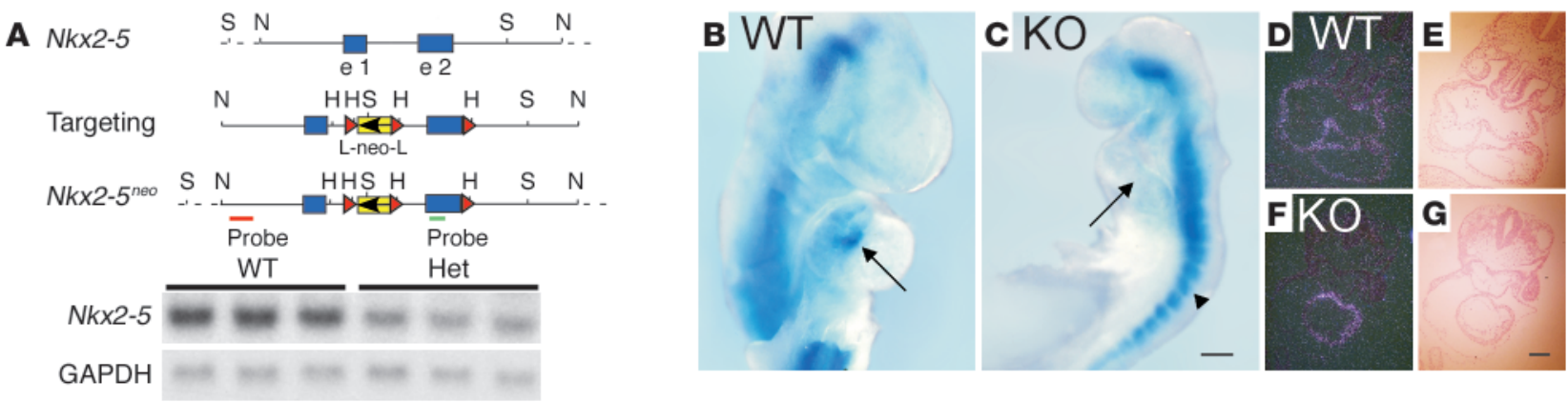

\section{Figure 1}

The absence of the AV node primordium in Nkx2-5-null mutant embryos. (A) Schematic diagram of the Nkx2-5 genomic structure, targeting construct, and Nkx2-5 neo allele and Northern blot analysis demonstrating a 50\% reduction in Nkx2-5 mRNA in the Nkx2-5+/neo adult ventricular myocardium. (B) WT Nkx2-5 E9.5 embryos that carry the minK-lacZ gene show blue X-gal staining in the inner curvature of the AV canal (arrow). (C) Nkx2-5 neo/neo E9.5 embryos show abnormal development of the heart tube and no minK-lacZ activity in the AV canal region where the AV node primordium is expected (arrow); staining is normal in the somites (arrowhead). In situ hybridization for minK mRNA and the corresponding brightfield images demonstrate expression throughout the myocardium of WT ( $\mathbf{D}$ and $\mathbf{E})$ and $N k \times 2-5^{\text {neo/neo }}(\mathbf{F}$ and $\mathbf{G})$ embryos. All images are representative of three or more embryos or mice. Comparisons of minK-lacZ expression were made between animals with identical copy numbers of the reporter allele. B and C show minK-lacZ homozygotes; heterozygotes yield similar results. WT, Nkx2-5 WT; Het, Nkx2-5+/neo; KO, Nkx2-5neo/neo; H, HindlII; N, Notl; S, Spel; e 1, exon; e 2, exon 2; L, loxP sequence; neo, pGK neomycin resistance cassette. Probe denotes fragments used for Southern blot analysis. Scale bars: $200 \mu \mathrm{m}$ (B and C), $100 \mu \mathrm{m}$ (D-G).

Nkx2-5neo/neo mice were recovered after E10, as was originally reported for two other $N k \times 2-5 \mathrm{KO}$ strains $(12,22)$. Progeny resulting from a double-heterozygous $\left(N k \times 2-5^{+/ n e o}-\mathrm{minK}^{+/ l a c z}\right)$ cross have $0-2$ copies of the minK-lacZ allele and the $N k \times 2-5$ WT allele. For the second independent set of conduction cell markers, we examined in the $N k \times 2-5^{+/-}$line the expression of $\mathrm{Cx} 40$, $\mathrm{Cx} 45(23,24) . N k \times 2-5^{+/-}$mice were not crossed to the minK-lacZ strain because both $\mathrm{KO}$ alleles were replaced with lacZ.

\section{Agenesis or hypoplasia of the central conduction system} in Nkx2-5 KO mice

The primordium of the AV node resides at the lesser curvature of the AV canal in the looping heart tube $(25,26)$. At E9.5, the cells in the region express minK-lacZ in Nkx2-5 WT embryos (Figure 1B). In contrast, E9.5 Nkx2-5neo/neo embryos show no cells that express minK-lacZ in the AV region (Figure 1C). The absence of marked cells suggests that the primordium is itself absent, but we cannot

\section{Figure 2}

Hypoplasia of the central conduction system in heterozygous Nkx2-5 KO mice. Histologic sections of adult hearts demonstrate the blue, $\mathrm{X}$-gal-stained cells of the AV node (A and $\mathbf{B})$ and $\mathrm{His}$ bundle ( $\mathbf{C}$ and D) in WT (A and $\mathbf{C}$ ) and Nkx2-5/neo (B and $\mathbf{D})$ hearts bearing one minK-lacZ allele. The histology of the AV node and His bundle were also examined by H\&E and immunohistochemical staining of WT $(\mathbf{E}, \mathbf{F}, \mathbf{I}, \mathbf{J})$ and $N k \times 2-5^{+/-}(\mathbf{G}, \mathbf{H}, \mathbf{K}, \mathbf{L})$ hearts. Cx40 (TRITC, red) and Cx43 (FITC, green) label conductive and contractile myocytes, respectively. The AV node $(\mathbf{F}$ and $\mathbf{H})$ and His bundle tissues ( $\mathbf{J}$ and $\mathbf{L}$ ) are circled. Consecutive sister sections from the same AV nodes were double-labeled for Cx40 (FITC, green) and Cx45 (TRITC, red) (circled in M and N). In the WT node (M), a small population of $\mathrm{C} \times 40^{+} / \mathrm{Cx} 45^{+}$cells is present (arrow), but a larger population bearing $\mathrm{Cx} 45$ alone predominates. In the $\mathrm{Nk} \times 2-5^{+/-}$node $(\mathbf{N})$ the $\mathrm{C} \times 40^{+} / \mathrm{C} \times 45^{+}$cells are present, but the population expressing Cx45 alone is absent. Images shown are representative of at least three separate animals. HET, Nkx2-5 $5^{+/-}$or Nkx2-5+/neo; AVN, AV node; His, His bundle. Scale bars: $100 \mu \mathrm{m}$.
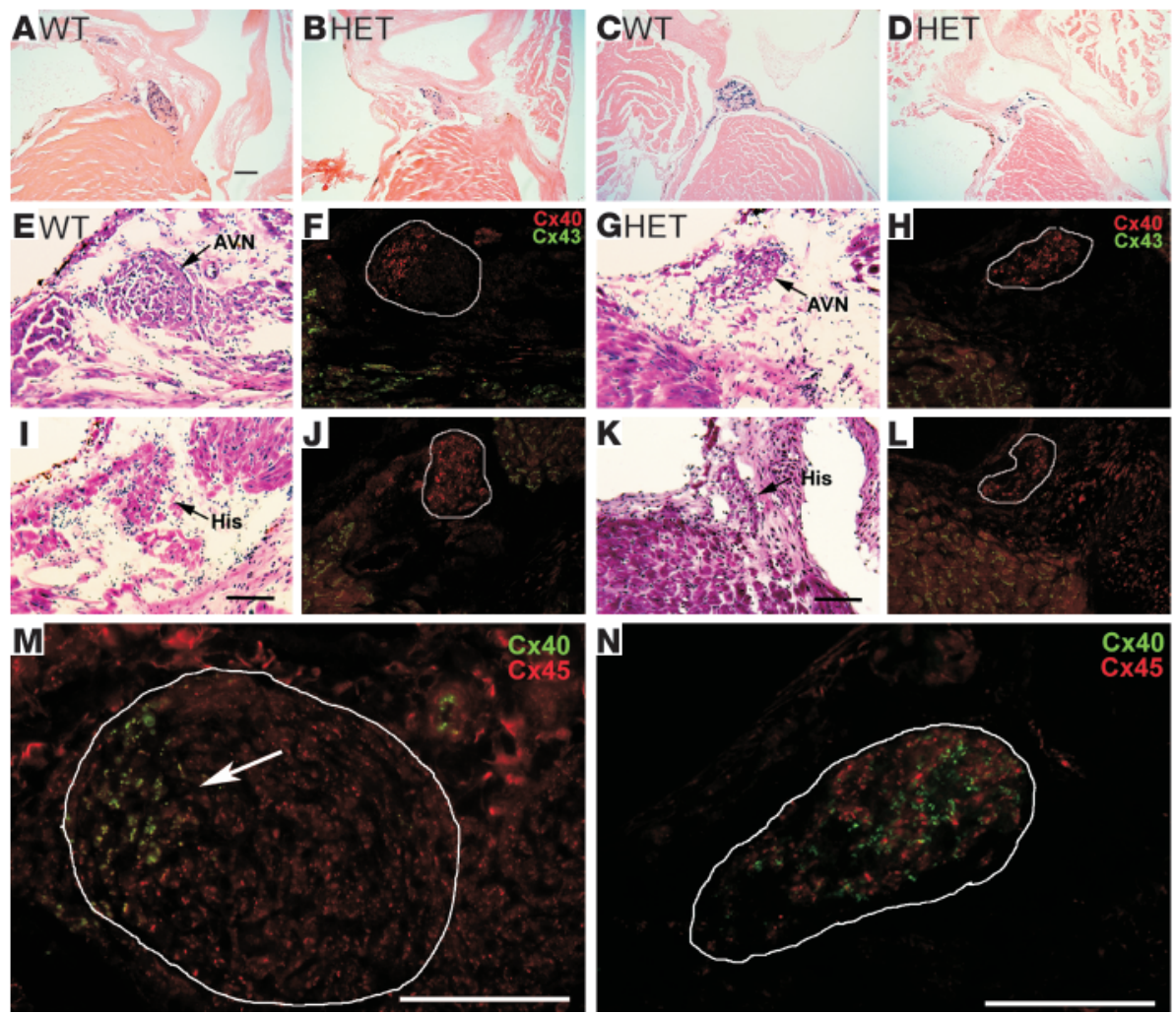
A

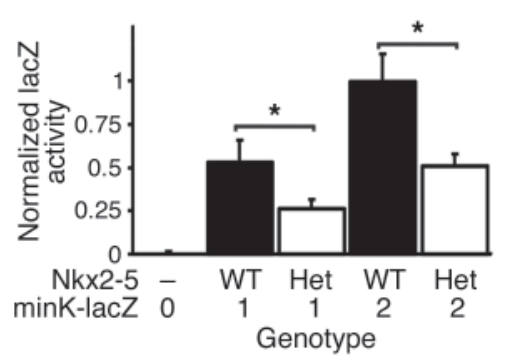

BWT

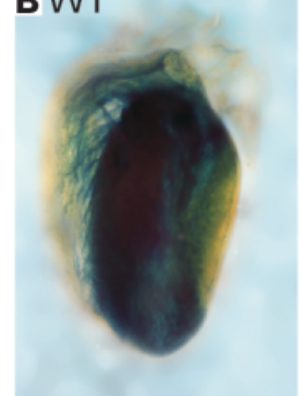

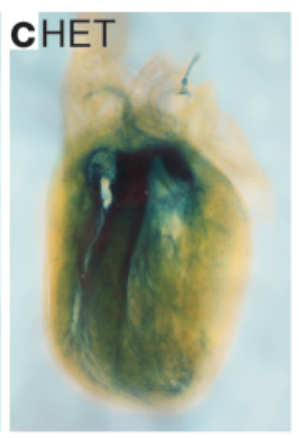

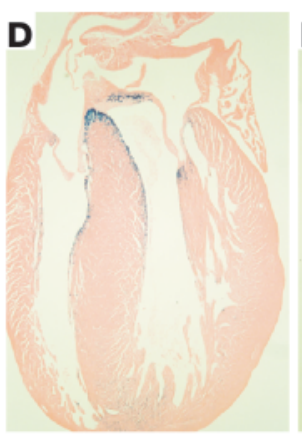

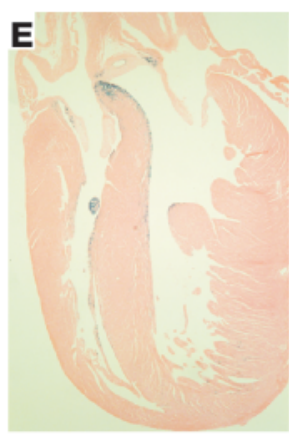

\section{Figure 3}

Hypoplasia of the peripheral conduction system in Nkx2-5neo/+ mice revealed by minK-lacZ expression. (A) In E14.5 hearts minK-lacZ enzymatic activity is proportional to minK-lacZ (copy numbers 0 , 1, or 2) and Nkx2-5 gene dosage. Whole-mount images of minK-lacZ-stained neonatal hearts contrast the dense Purkinje fiber network in WT (B and D) compared with the hypocellular system in Nkx2-5+/neo mice (C and E). Sections show that the prominent blue X-gal stain shown in the whole mount is the peripheral conduction system at the interventricular septum (D and $\mathbf{E}$ ). The hearts shown were from minK-lacZ homozygotes; heterozygotes yield similar results. The images shown are representative of at least three hearts. $P<0.05$.

exclude the possibility that the primordial AV node cells uniquely require Nkx2-5 for transcription of the minK-lacZ gene. Two observations suggest that transcription at the minK locus does not require $\mathrm{Nkx2}-5$ in general. First, the somites, which lack Nkx2-5, express minK-lacZ. Second, minK mRNA is expressed throughout the myocardium of both WT (Figure 1, D and E) and Nkx2-5 neo/neo embryos (Figure 1, F and G), as previously described (27).
Nkx2-5 haploinsufficient mice form a smaller central conduction system. When viewed in frontal (four-chamber) histologic sections, the $\mathrm{AV}$ node is located posterior to and immediately above the interventricular septum (IVS). The His bundle extends anteriorly from the AV node along the crest of the IVS. The AV node and His bundle express minK-lacZ and $\mathrm{Cx}_{40}$ and $\mathrm{Cx} 45$. Serial sections through adult hearts demonstrate that the $\mathrm{AV}$ node and His bun-

\section{Figure 4}

Cx40 immunohistochemistry confirms hypoplasia of the peripheral Purkinje system and normal cellular

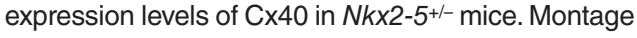
confocal images demonstrate $\mathrm{Cx} 40$ expression as red, punctate staining in the subendocardial Purkinje fibers of WT (A and $\mathbf{C}$ ) and $N k \times 2-5^{+/-}$(B and $\left.\mathbf{D}\right)$ left ventricular myocardium. The distribution of Cx40 in

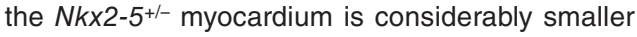
than in WT (arrows in A and $\mathbf{B}$ ). Higher magnification images of the boxed areas in $\mathbf{A}$ and $\mathbf{B}$ reveal the increased thickness of Purkinje fiber layers (arrowheads) in the WT (C) compared with Nkx2-5 $5^{+-}$heart (D). LV, left ventricle. Scale bars: $100 \mu \mathrm{m}$. (E) Cell counts within Cx40-positive domains reveal that Purkinje cell numbers are reduced by approximately half within sections of $N k \times 2-5^{+/-}$ventricles $(P<0.05)$. (F) Nkx2-5+/- and WT Purkinje cells contain approximately the same the number of $\mathrm{Cx} 40$ particles per cell. Three hearts each from WT and Nkx2-5+- animals were examined.

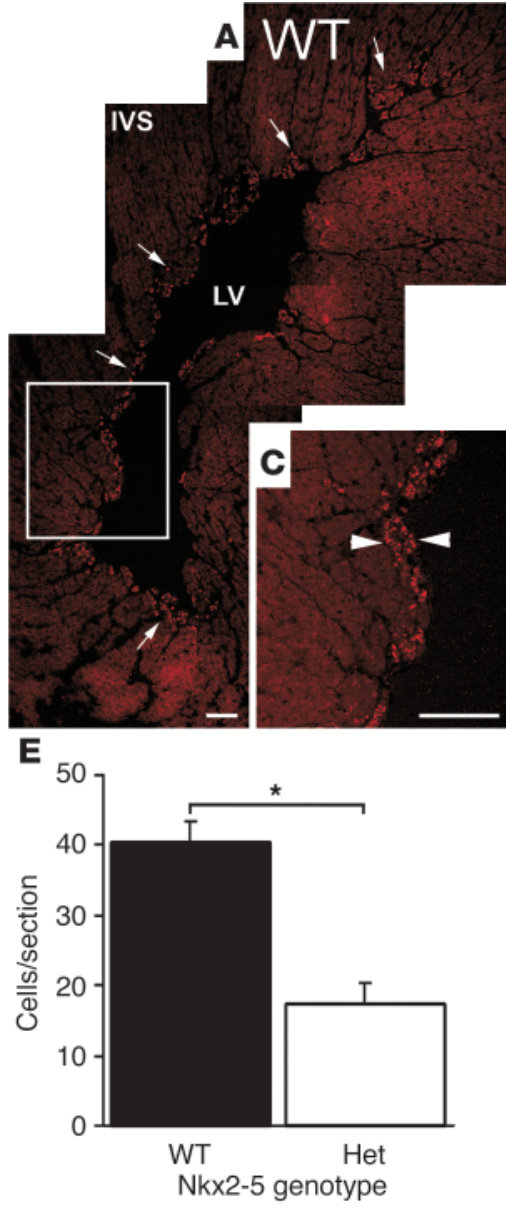

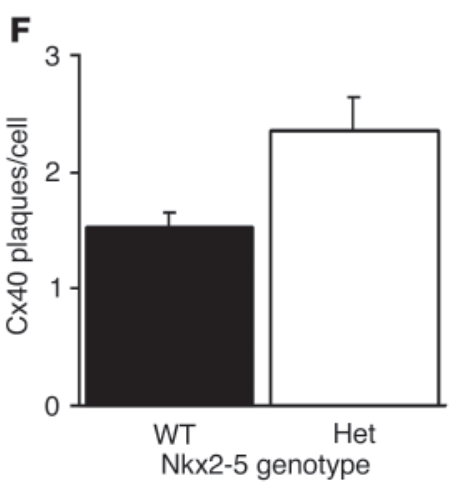



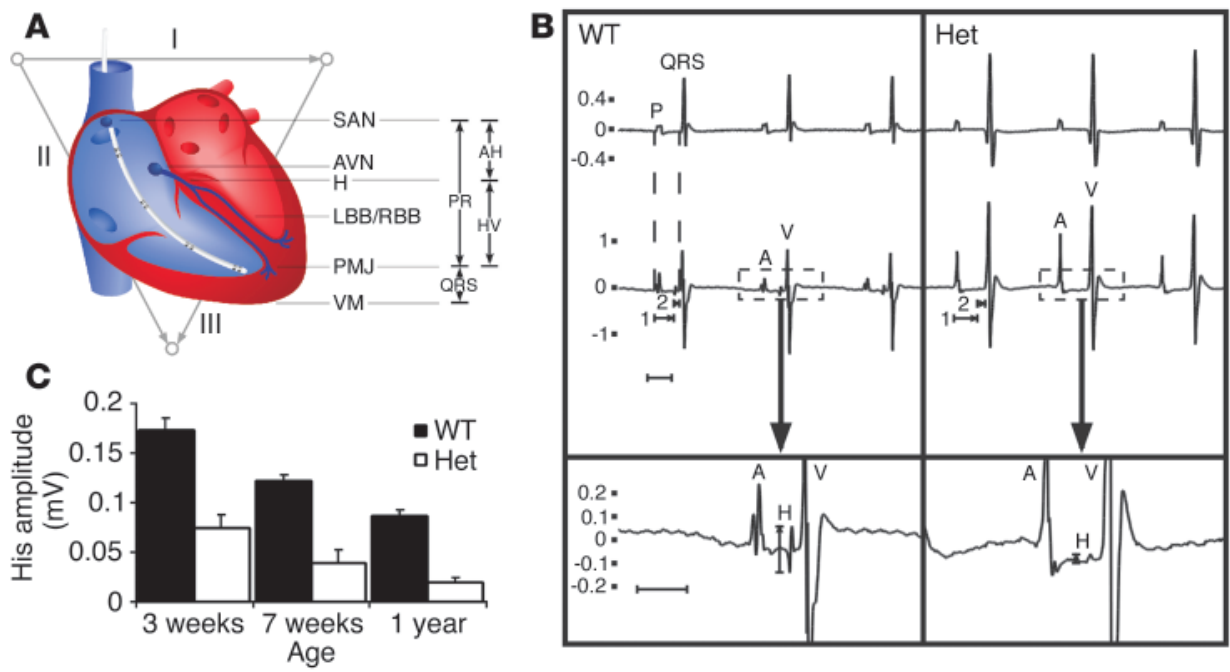

Figure 5

ECG correlates of anatomic structures in the cardiac conduction system in WT and Nkx2-5+/- mice. (A) Schematic diagram of the cardiac conduction system and ECG intervals as symbolized by the surface vectors I, II, and III and the intracardiac catheter. (B) Representative surface and intracardiac electrogram from a WT and $\mathrm{Nk} \times 2-5^{+-}$mouse. The upper and middle tracings are simultaneous surface ECGs and IEGMs. The lower tracing shows the intracardiac recording at higher magnification and demonstrates the difference in His bundle signal amplitude between WT and Nkx2-5+/- mice. Atrial (P, A) and ventricular (QRS, V) depolarizations are depicted. The IEGM shows $\mathrm{His}(\mathrm{H})$ depolarization. Atrio-His and His-ventricular intervals are denoted (1 and 2). Note that ventricular depolarization or QRS interval begins immediately after the HV interval. (C) The amplitude of His depolarization is diminished in Nkx2-5+/- mice at all ages examined $(P<0.0001$ in all age groups). AV, atrial-ventricular; $\mathrm{AH}$, atrio-His; HV, His-ventricular; LBB/RBB, left and right bundle branch; PMJ, Purkinje-myocyte junction; SAN, sinoatrial node; VM, ventricular myocardium. compared with the $N k \times 2-5^{+/-}$mice (Figure 2, G-L).

The normal mouse AV nodal region contains two cell populations or compartments as defined by gap junction isoform expression (28). The proximal, posterior compartment expresses $\mathrm{Cx} 45$ but not $\mathrm{Cx} 40$. The distal, anterior compartment, which connects to the His bundle, expresses both $\mathrm{Cx} 40$ and $\mathrm{Cx} 45$. In the WT, there is a discrete fascicular structure that contains a smaller $\mathrm{Cx} 40^{+} / \mathrm{Cx} 45^{+}$and a larger $\mathrm{Cx} 40^{-} / \mathrm{Cx} 45^{+}$compartment (Figure 2M). The Nkx2-5 $5^{+-}$node has only the distal $\mathrm{Cx} 40^{+} / \mathrm{Cx} 45^{+}$compartment (Figure $2 \mathrm{~N}$ ). The proximal $\mathrm{Cx} 40^{-} / \mathrm{Cx} 45^{+}$compartment is absent or severely attenuated. Qualitatively similar levels of $\mathrm{Cx} 40$ and $\mathrm{Cx} 45$ were present in the remaining $\mathrm{Cx} 40^{+} / \mathrm{Cx} 45^{+}$ compartment of $\mathrm{Nk} \times 2-5^{+/-}$and WT mice. The $\mathrm{Cx} 40^{+} / \mathrm{Cx} 45^{+}$region reveals a high degree of overlap of the immunofluorescent green $\mathrm{Cx} 40$ (FITC) and red Cx45 (TRITC) signals, yielding a yellow signal when merged. The colocalization indicates that a cell in the AV node can express both connexin isoforms and that the two dle in $N k \times 2-5^{+/ n e o}$ mice are smaller and have fewer minK-lacZ ${ }^{+}$cells than the WT mice (Figure 2, A and B). Cross sections of the His bundle demonstrate the size difference most clearly. Compare the wispy strand of the Nkx2-5+/neo heart to the wide bundle of the WT at the crest of the ventricular septum where the bundle branches first diverge (Figure 2, C and D). Cx40 expression similarly delineated the larger AV node and His bundle of the WT (Figure 2, E-J) connexins are usually present in the same gap-junctional plaques, as previously reported (28).

\section{Hypocellularity of the peripheral conduction system in heterozygous $\mathrm{Nk} \times 2-5 \mathrm{KO}$ mice}

By E14 the peripheral conduction system has developed (29). Although Nkx2-5-null mutant embryos die around E10, the het-

Table 1

Cardiac conduction intervals in Nkx2-5+/- and WT mice

\begin{tabular}{|c|c|c|c|c|c|c|c|c|}
\hline & \multicolumn{4}{|c|}{ ECG } & \multicolumn{4}{|c|}{ IEGM } \\
\hline & HR (bpm) & PR (ms) & QRS (ms) & QTm (ms) & HR (bpm) & $\mathrm{AH}(\mathrm{ms})$ & $\mathrm{HV}$ (ms) & AVI (ms) \\
\hline \multicolumn{9}{|l|}{3 weeks old } \\
\hline Het $(20,8)$ & $333 \pm 10$ & $38.2 \pm 0.9$ & $15.0 \pm 0.4$ & $22.0 \pm 0.6$ & $284 \pm 8$ & $30.1 \pm 0.9$ & $10.1 \pm 0.5$ & $40.2 \pm 1.2$ \\
\hline WT (18, 12 mice) & $310 \pm 12$ & $39.0 \pm 1.0$ & $13.4 \pm 0.4$ & $21.2 \pm 0.5$ & $271 \pm 16$ & $32.2 \pm 1.5$ & $11.2 \pm 0.3$ & $43.4 \pm 1.5$ \\
\hline$P$ values & NS & NS & 0.01 & NS & NS & NS & NS & NS \\
\hline \multicolumn{9}{|l|}{7 weeks old } \\
\hline Het $(13,11)$ & $345 \pm 11$ & $44.4 \pm 1.0$ & $15.7 \pm 0.7$ & $19.3 \pm 0.7$ & $320 \pm 19$ & $44.0 \pm 3.3$ & $10.5 \pm 0.6$ & $54.5 \pm 3.2$ \\
\hline WT $(9,9)$ & $357 \pm 11$ & $39.8 \pm 1.1$ & $12.9 \pm 0.3$ & $19.7 \pm 0.8$ & $327 \pm 17$ & $34.6 \pm 1.4$ & $10.3 \pm 0.6$ & $44.9 \pm 1.4$ \\
\hline$P$ values & NS & 0.006 & 0.003 & NS & NS & 0.03 & NS & 0.02 \\
\hline \multicolumn{9}{|l|}{1 year old } \\
\hline Het $(21,9)$ & $358 \pm 15$ & $50.0 \pm 1.4$ & $17.0 \pm 0.4$ & $25.4 \pm 0.5$ & $319 \pm 18$ & $45.2 \pm 1.9$ & $11.7 \pm 0.6$ & $56.9 \pm 1.5$ \\
\hline WT $(20,20)$ & $373 \pm 10$ & $44.6 \pm 1.2$ & $12.5 \pm 0.3$ & $24.4 \pm 0.4$ & $338 \pm 8$ & $36.0 \pm 1.0$ & $11.8 \pm 0.4$ & $47.9 \pm 1.2$ \\
\hline$P$ values & NS & 0.004 & $<0.001$ & NS & NS & 0.0001 & NS & 0.0005 \\
\hline
\end{tabular}


Table 2

Impaired atrioventricular conduction in Nkx2-5+- mice

\begin{tabular}{|c|c|c|c|}
\hline & \multicolumn{3}{|c|}{ AV node functional intervals } \\
\hline & 1:1 (ms) & 2:1 (ms) & AVERP (ms) \\
\hline \multicolumn{4}{|c|}{3 weeks old } \\
\hline Het (9) & $125.0 \pm 5.1$ & $100.6 \pm 3.8$ & $97.8 \pm 6.9$ \\
\hline WT (12) & $123.3 \pm 4.4$ & $97.1 \pm 3.3$ & $87.7 \pm 3.8$ \\
\hline$P$ values & NS & NS & NS \\
\hline \multicolumn{4}{|c|}{7 weeks old } \\
\hline Het (12) & $132.9 \pm 3.8$ & $103.3 \pm 2.7$ & $98.6 \pm 3.3$ \\
\hline WT (9) & $118.9 \pm 3.0$ & $91.7 \pm 2.4$ & $85.6 \pm 3.7$ \\
\hline$P$ values & 0.01 & 0.01 & 0.02 \\
\hline \multicolumn{4}{|c|}{1 year old } \\
\hline Het (21) & $128.3 \pm 3.6$ & $93.3 \pm 3.0$ & $92.8 \pm 4.1$ \\
\hline WT (20) & $116.3 \pm 2.7$ & $86.7 \pm 2.7$ & $76.0 \pm 3.1$ \\
\hline$P$ values & 0.002 & 0.03 & $<0.001$ \\
\hline \multicolumn{4}{|c|}{$\begin{array}{l}\text { Rapid atrial pacing reveals diminished AV node function in } N k \times 2-5^{+/-} \\
\text {compared with WT mice. The } 1: 1 \text { and } 2: 1 \text { cycle lengths are the minimal } \\
\text { and maximal right atrial pacing intervals in which the ventricular beat fol- } \\
\text { lows each paced beat at a constant interval or skips every other beat, } \\
\text { respectively. To determine AVERP, the right atrium is given a train of } \\
\text { eight beats (S1, } 150 \text {-ms cycle length) followed by a single beat (S2) at } \\
\text { incrementally shorter intervals after the last S1. AVERP is the S1S2 } \\
\text { interval in which the AV node fails to propagate the S2 beat. Data are } \\
\text { presented as mean plus or minus SEM. Het, } N k \times 2-5^{+/-} \text {. }\end{array}$} \\
\hline
\end{tabular}

erozygotes survive to birth. For an initial assessment of the conduction system in E14.5 WT and Nkx2-5+/neo embryos we measured minK-lacZ activity in whole-heart lysates. As expected, homozygous minK-lacZ embryos had twice as much lacZ activity as heterozygotes given equivalent $N k \times 2-5$ gene dosages. $N k \times 2-5$ haploinsufficiency was also associated with a proportionate reduction in lacZ activity (Figure 3A). Hypocellularity of the peripheral Purkinje network in Nkx2$5^{+/ n e o}$ hearts is largely the cause of the reduced enzymatic activity. Compare the dense blue staining of the $\mathrm{AV}$ ring and interventricular septum of newborn WT mice (Figure 3, B and D) to the sparser network in the Nkx2-5+neo heart (Figure 3, C and E). Hence, individual fibers of the left and right bundle branch radiations can be discerned in the mutant (Figure 3C) but not the WT mouse (Figure 3B).

Quantification of Purkinje cells in WT and Nkx2-5 $5^{+/}$hearts was obtained by $\mathrm{Cx} 40$ immunohistochemistry. Distinct subendocardial clusters of $\mathrm{Cx} 40^{+}$myocytes, corresponding to Purkinje cells, were located in the ventricular septum. Nkx2-5 $5^{+-}$hearts had fewer fibers, each of which had fewer cells when compared with WT hearts (Figure 4, A-D). $N k \times 2-5^{+/-}$ventricles had approximately half as many $\mathrm{Cx} 40^{+}$Purkinje cells as the WT (Figure 4E), which parallels the quantitative reduction in minK-lacZ activity in E14.5 Nkx2-5neo/+ mice (Figure 3A). The discrete plaquelike structure and near crystalline packing of gap junction channels within a plaque permit quantification of connexins by immunofluorescent confocal microscopy (30). Surprisingly, no difference in the number of Cx40 plaques per Purkinje cell was noted between $N k \times 2-5^{+/-}$and WT (Figure 4F). Cx40 plaque size and fluorescence intensity levels were comparable between the WT and Nkx2-5+/- cells (data not shown).

\section{Conduction and electrophysiologic abnormalities in Nkx2-5 haploinsufficiency}

Propagation of the heartbeat through the conduction system can be followed by surface ECG and IEGM (Figure 5). The hypoplas- tic central and peripheral conduction systems in $N k \times 2-5 \mathrm{KO}$ mice were associated with physiologic abnormalities at each affected level. ECG measurements were obtained in mice ranging from 3 weeks to 1 year of age. Nkx2-5 showed similar conduction and electrophysiologic abnormalities (data not shown). Ventricular repolarization, as determined by the rate-corrected QT interval (31), was the same in $N k \times 2-5^{+/-}$and WT mice (Table 1).

The PR interval reflects conduction from the sinus node to the onset of ventricular myocardial activation. The PR is composed of the intracardiac $\mathrm{AH}$ and $\mathrm{HV}$ intervals, corresponding to conduction from the atrium to the His bundle and from the His bundle to the Purkinje-ventricular myocyte junction. $N k \times 2-5^{+/-}$mice have a prolonged PR interval at age 7 weeks and older (Table 1). The prolonged PR interval in $N k \times 2-5^{+/-}$mice resulted from prolongation of the AH interval. Because the durations of the $\mathrm{P}$ wave were equal in WT and Nkx2-5 $5^{+/}$mice, which indicates similar atrial conduction velocities, the prolonged PR must result from a defect in the AV node. Nkx2-5+/- mice, age 7 weeks and older, also have longer 1:1 and 2:1 cycle lengths and AV node effective refractory periods than the WT as determined by rapid atrial pacing. The differences further demonstrate diminished $\mathrm{AV}$ node function in $N k \times 2-5^{+/-}$mice (Table 2). Nkx2-5 $5^{+/-}$mice as old as 2 years do not progress beyond first-degree AV block (data not shown). Curiously, 3-week-old $N k \times 2-5^{+/-}$mice have a normal PR interval and AV node function as assessed by atrial pacing. Autonomic influences are unlikely to obscure any intrinsic cardiac defect because $\beta$-adrenergic and cholinergic blockade did not elicit a difference between the WT and heterozygous 3-week-old mice (data not shown).

The His signal amplitude on the IEGM was markedly diminished in Nkx2-5 haploinsufficient mice as young as 3 weeks, when they were large enough to permit intravenous insertion of the electrode catheter. All WT mice had an easily detected His signal, whereas all Nkx2-5 haploinsufficient mice had a small or undetectable signal (Figure 5). The difference was so clear that the Nkx2-5 genotype could be predicted by visual inspection of the His signal in mice of any age. For example, the genotype was correctly determined by a blinded observer in all 57 one-year-old mice based solely on the His signal $\left(21 \mathrm{Nk} \times 2-5^{+/-}, 14 \mathrm{Nk} \times 2-5^{+/ n e o}, 22 \mathrm{Nk} \times 2-5^{+/+}\right)$. Normalization for the amplitude of the atrial or ventricular electrogram did not affect the results (data not shown.)

The HV interval, measured between the initial deflections of the His and ventricular signals, predominantly reflects conduction through the His-Purkinje system to the Purkinje-myocyte junction in the right ventricular apex. WT and $N k \times 2-5^{+/-}$mice had similar intervals at all ages (Table 1). Therefore, the conduction velocities of WT and Nkx2-5+/- Purkinje fibers are the same.

The QRS interval, which continues after the HV, represents depolarization of the entire ventricular myocardium. Depolarization begins at the Purkinje-myocyte junction and ends at the myocyte farthest from a terminal Purkinje-myocyte junction. Nkx2-5 at all ages showed a significantly prolonged QRS interval (Table 1).

\section{Discussion}

Mutations of Nkx2-5 cause atrioventricular conduction defects in humans and a mouse KO model. The current work suggests an anatomic basis for conduction defects present in Nkx2-5 loss of function. $N k \times 2-5$ gene dosage affects the number of myocytes in the murine conduction system. Null mutant embryos entirely lack the minK-lacZ-expressing cells in the lesser curvature of the heart that 
are probably the progenitors of the AV node. Haploinsufficient embryos and adults have cellular hypoplasia of the central and peripheral conduction systems, as marked by minK-lacZ or Cx 40 and $\mathrm{Cx} 45$ expression. In addition, a group of $\mathrm{Cx} 40^{-} / \mathrm{Cx} 45^{+}$cells is strikingly absent in the Nkx2-5+- AV node. Thus, either Nkx2-5 mutant embryos recruit fewer myocytes into the conduction system, or myocytes are lost after recruitment, perhaps by apoptosis. If $\mathrm{Nkx} 2-5$ affects recruitment, then $\mathrm{Nkx}$-5 might regulate specification of a small population of recruiting cells or a component of an inductive signaling pathway. Diminished proliferation of conduction cells probably cannot explain hypoplasia in the Nkx2-5 KO mouse because the myocytes exit the cell cycle during recruitment $(2,4)$.

The physiologic data show that heterozygous Nkx2-5 KO mice have defects in the AV node, His bundle, and intraventricular conduction. The results differ from a previous report in which only PR prolongation was found in a specific strain and sex of Nkx2-5 KO mice (32). One potential explanation is that the high temporal resolution $(1 \mathrm{~ms})$ in this study permitted detection of interval differences relevant to the rapid murine heart rate.

The His bundle demonstrates the clearest relationship between structural and functional defects in the heterozygous $N k \times 2-5$ mutant conduction system. Heterozygous Nkx2-5 KO mice have thin His bundles, as defined by either minK-lacZ or Cx40 expression. Intracardiac electrogram consistently demonstrate a low amplitude signal of His depolarization in the heterozygous $\mathrm{KO}$ mice at any age, which is most directly explained by the wispiness of the bundle. We have not observed any consistent difference in the anatomy or structure of the atrioventricular septum that could affect the course of the electrode catheter or its ability to measure the His bundle signal in Nkx2-5 mutant mice. Diminished cellular depolarization in the His bundle is possible, but this would only compound the effect of a thin His bundle.

A second direct relationship can be drawn between the low density of peripheral Purkinje cells and the prolonged QRS interval in Nkx2-5 KO mice. The low density of Purkinje cells is suggested by comparison of minK-lacZ expression in WT and $N k \times 2-5^{+/ n e o}$ hearts and confirmed by direct counts of $\mathrm{Cx} 40^{+}$ myocytes. The hypocellular Purkinje network in heterozygous Nkx2-5 KO mice could cause prolongation of the QRS interval because each terminal Purkinje cell must depolarize a larger region of contractile myocardium where conduction is slow relative to the Purkinje fibers.

A seeming paradox arises from the prolonged QRS in the presence of a normal HV interval in the Nkx2-5 mutant mice. Based on the definitions of the QRS and HV intervals, however, the observation is consistent with the fact that conduction velocity through a Purkinje fiber and the number of terminal Purkinje fibers that depolarize the ventricles are separate variables. The HV interval, which largely represents conduction through the His-Purkinje system, indicates that individual Purkinje fibers in the Nkx2-5 mutant mouse conduct normally. The QRS extends beyond the HV to include ventricular depolarization after the Purkinje-myocyte junction. We suggest that the prolonged QRS results from the decreased ratio of terminal Purkinje cells to contractile myocardial volume in Nkx2-5 mutant hearts. The prolonged QRS cannot be attributed to the downregulation of $\mathrm{Cx} 43$, the major gap junction isoform in the contractile myocardium, because it is normally expressed in $N k \times 2-5^{+/-}$mice (33).

Interestingly, the size of the region ultimately depolarized by each terminal Purkinje cell can explain QRS durations across species with large and small hearts. For example, humans and mice have a predominantly subendocardial distribution of Purkinje fibers. As would be predicted, the QRS durations in humans and mice, about 90 and $12 \mathrm{~ms}$, respectively, scale with the thicknesses of their left ventricular wall, approximately 9 and $1 \mathrm{~mm}$. In contrast, the bovine heart is four times larger than the human heart, but the QRS durations are similar, probably because the bovine Purkinje network extends from the subendocardium almost to the epicardium (34). Prolonged QRS intervals have not been described in human Nkx2-5 mutation, but the small interval differences present in mutant mice might be subclinical in patients for whom recognition of larger prolongations are more relevant, such as complete right bundle branch block.

The relationship between a small $\mathrm{AV}$ node that lacks $\mathrm{Cx} 40^{-} / \mathrm{Cx} 45^{+}$ cells and prolongation of the PR or AH intervals in Nkx2-5 mutant mice is less clear because the onset of PR prolongation and AV nodal functional defects, that is, longer-paced 1:1 and 2:1 block cycle lengths and increased AVERP, occurs between 3 and 7 weeks of age. As WT mice age, the PR interval prolongs independently of the heart rate. The mechanistic basis is unknown, but maturational changes of $\mathrm{AV}$ node physiology in association with hypoplasia or absence of the $\mathrm{Cx} 40^{-} / \mathrm{Cx} 45^{+}$cell population could cause the atrioventricular conduction defect present in the Nkx2-5 mutant mice. For example, changes in the cellular distribution of gap junctions have been described in the terminal crest from the right atrium of neonatal, weanling, and adult rabbits (35).

A limitation of the current work is that we have not excluded changes in every protein that participates in the action potential. Still, the downregulation of $\mathrm{Cx} 40$, the predominant gap junction protein in the conduction system, does not seem to occur in the conduction system of Nkx2-5 heterozygotes and hence cannot cause the prolonged PR and QRS intervals, as could be reasonably supposed (36). Homozygous $\mathrm{Cx} 40 \mathrm{KO}$ mice have slow conduction through the Purkinje system and Purkinje-myocyte junction that manifests as prolonged HV and QRS intervals $(37,38)$. Heterozygous $\mathrm{Cx} 40 \mathrm{KO}$ mice have normal conduction despite having halfnormal $\mathrm{Cx} 40$ protein levels, indicating that a greater than $50 \%$ reduction is necessary to cause a conduction defect (39). In Nkx2-5/and WT mice the number of $\mathrm{Cx} 40$ particles per Purkinje cell is the same, consistent with their equivalent $\mathrm{HV}$ intervals. The expression of $\mathrm{Cx} 40$ and $\mathrm{Cx} 45$ in the $\mathrm{Cx} 40^{+} / \mathrm{Cx}_{4} 45^{+}$compartment of the AV node likewise appears similar. The observations by no means disprove the transcriptional regulation of $\mathrm{Cx} 40$ by $\mathrm{Nkx} 2-5$ but do demonstrate that half-normal gene dosage is sufficient for specific expression of $\mathrm{Cx} 40$ in the conduction system.

In summary, the number of myocytes that ultimately become conduction cells depends upon Nkx2-5 gene dosage. Hypoplasia of anatomic structures in the conduction system of heterozygous $N k \times 2-5$ $\mathrm{KO}$ mice likely contributes to diminished AV node function and PR prolongation and can completely explain electrophysiologic defects such as a small His bundle-depolarization signal and intraventricular conduction delay. For the His bundle and peripheral Purkinje system, it is unnecessary to invoke a role for Nkx2-5 in the transcriptional regulation of a channel or gap junction such as $\mathrm{Cx} 40$.

\section{Acknowledgments}

We thank Olga Rozhitskaya, Maria Rivera, Laura Barbosky, and Daniel Iaun-bor Chen for their assistance. J. Barhanin kindly provided the minK in situ probe. This work is supported by grants from the NIH (to P.Y. Jay, R.G. Gourdie, and S. Izumo), the Marram and Carpenter Fund for Innovation and the Charles H. Hood Founda- 
tion (to P.Y. Jay), and the Deutsche Akademie der Naturforscher Leopoldina (to A. Buerger).

Received for publication August 20, 2003, and accepted in revised form February 17, 2004.

1. Wang, G.F., and Stockdale, F.E. 1999. Chamber-specific gene expression and regulation during heart development. In Heart development. R.P. Harvey and N. Rosenthal, editors. Academic Press. San Diego, California, USA. 357-369.

2. Cheng, G., et al. 1999. Development of the cardiac conduction system involves recruitment within a multipotent cardiomyogenic lineage. Development. 126:5041-5049.

3. Gourdie, R.G., Mima, T., Thompson, R.P., and Mikawa, T. 1995. Terminal diversification of the myocyte lineage generates Purkinje fibers of the cardiac conduction system. Development. 121:1423-1431.

4. Sedmera, D., et al. 2003. Spatiotemporal pattern of commitment to slowed proliferation in the embryonic mouse heart indicates progressive differentiation of the cardiac conduction system. Anat. Rec. 274A:773-777.

5. Gourdie, R.G., Wei, Y., Kim, D., Klatt, S.C., and Mikawa, T. 1998. Endothelin-induced conversion of embryonic heart muscle cells into impulse-conducting Purkinje fibers. Proc. Natl. Acad. Sci. U. S. A. 95:6815-6818.

6. Rentschler, S., et al. 2002. Neuregulin-1 promotes formation of the murine cardiac conduction system. Proc. Natl. Acad. Sci. U. S. A. 99:10464-10469.

7. Askanase, A.D., et al. 2002. Spectrum and progression of conduction abnormalities in infants born to mothers with anti-SSA/Ro-SSB/La antibodies. Lupus. 11:145-151.

8. Jay, P.Y., Powell, A.J., Sherwood, M.C., and Izumo, S. 2003. Nkx2-5 and congenital heart disease. In The molecular basis of inborn errors of development. C.J. Epstein, R.P. Erickson, and A. Wynshaw-Boris, editors. Oxford University Press. New York, New York, USA. 607-614.

9. Schott, J.J., et al. 1998. Congenital heart disease caused by mutations in the transcription factor NKX2-5. Science. 281:108-111.

10. Benson, D.W., et al. 1999. Mutations in the cardiac transcription factor NKX2.5 affect diverse cardiac developmental pathways. J. Clin. Invest. 104: $1567-1573$.

11. Thomas, P.S., et al. 2001. Elevated expression of $\mathrm{Nkx}-2.5$ in developing myocardial conduction cells. Anat. Rec. 263:307-313.

12. Tanaka, M., Chen, Z., Bartunkova, S., Yamasaki, N., and Izumo, S. 1999. The cardiac homeobox gene Csx/Nkx2.5 lies genetically upstream of multiple genes essential for heart development. Development. 126:1269-1280.

13. Kupershmidt, S., et al. 1999. Replacement by homologous recombination of the minK gene with lac $Z$ reveals restriction of minK expression to the
Address correspondence to: Patrick Jay, Department of Cardiology, Children's Hospital and Department of Pediatrics, Harvard Medical School, 300 Longwood Avenue, Boston, Massachusetts 02115, USA. Phone: (617) 355-8571; Fax: (617) 975-5268; E-mail: patrick.jay@childrens.harvard.edu. mouse cardiac conduction system. Circ. Res. 84:146-152.

14. Coppen, S.R., Dupont, E., Rothery, S., and Severs, N.J. 1998. Connexin45 expression is preferentially associated with the ventricular conduction system in mouse and rat heart. Circ. Res. 82:232-243.

15. Barker, R.J., Price, R.L., and Gourdie, R.G. 2002. Increased association of $\mathrm{ZO}-1$ with connexin 43 during remodeling of cardiac gap junctions. Circ. Res. 90:317-324.

16. Maguire, C.T., Bevilacqua, L.M., Wakimoto, H., Gehrmann, J., and Berul, C.I. 2000. Maturational atrioventricular nodal physiology in the mouse. J. Cardiovasc. Electrophysiol. 11:557-564.

17. Berul, C.I., et al. 2001. Ventricular arrhythmia vulnerability in cardiomyopathic mice with homozygous mutant myosin-binding protein $\mathrm{C}$ gene. Circulation. 104:2734-2739.

18. Berul, C.I., Aronovitz, M.J., Wang, P.J., and Mendelsohn, M.E. 1996. In vivo cardiac electrophysiology studies in the mouse. Circulation. 94:2641-2648.

19. Kondo, R.P., Anderson, R.H., Kupershmidt, S., Roden, D.M., and Evans, S.M. 2003. Development of the cardiac conduction system as delineated by minK-lacZ. J. Cardiovasc. Electrophysiol. 14:383-391.

20. Gage, P.J., Suh, H., and Camper, S.A. 1999. Dosage requirement of Pitx2 for development of multiple organs. Development. 126:4643-4651.

21. Meyers, E.N., Lewandoski, M., and Martin, G.R. 1998. An Fgf8 mutant allelic series generated by Cre- and Flp-mediated recombination. Nat. Genet. 18:136-141.

22. Lyons, I., et al. 1995. Myogenic and morphogenetic defects in the heart tubes of murine embryos lacking the homeo box gene Nkx2-5. Genes Dev. 9:1654-1666.

23. Gourdie, R.G., et al. 1993. The spatial distribution and relative abundance of gap-junctional connexin 40 and connexin 43 correlate to functional properties of components of the cardiac atrioventricular conduction system. J. Cell Sci. 105:985-991.

24. Gourdie, R.G., Green, C.R., Severs, N.J., Anderson, R.H., and Thompson, R.P. 1993. Evidence for a distinct gap-junctional phenotype in ventricular conduction tissues of the developing and mature avian heart. Circ. Res. 72:278-289.

25. Viragh, S., and Challice, C.E. 1977. The development of the conduction system in the mouse embryo heart. II. Histogenesis of the atrioventricular node and bundle. Dev. Biol. 56:397-411.

26. Viragh, S. and Challice, C.E. 1977. The development of the conduction system in the mouse embryo heart. I. The first embryonic A-V conduction pathway. Dev. Biol. 56:382-396.
27. Franco, D., et al. 2001. Divergent expression of delayed rectifier $\mathrm{K}(+)$ channel subunits during mouse heart development. Cardiovasc. Res. 52:65-75.

28. Coppen, S.R., Severs, N.J., and Gourdie, R.G. 1999. Connexin45 (alpha 6) expression delineates an extended conduction system in the embryonic and mature rodent heart. Dev. Genet. 24:82-90.

29. Viragh, S., and Challice, C.E. 1982. The development of the conduction system in the mouse embryo heart. IV. Differentiation of the atrioventricular conduction system. Dev. Biol. 89:25-40.

30. Green, C.R., Peters, N.S., Gourdie, R.G., Rothery, S., and Severs, N.J. 1993. Validation of immunohistochemical quantification in confocal scanning laser microscopy: a comparative assessment of gap junction size with confocal and ultrastructural techniques. J. Histochem. Cytochem. 41:1339-1349.

31. Mitchell, G.F., Jeron, A., and Koren, G. 1998. Measurement of heart rate and Q-T interval in the conscious mouse. Am. J. Physiol. 274:H747-H751.

32. Biben, C., et al. 2000. Cardiac septal and valvular dysmorphogenesis in mice heterozygous for mutations in the homeobox gene Nkx2-5. Circ. Res. 87:888-895.

33. Tanaka, M., et al. 2002. A mouse model of congenital heart disease: cardiac arrhythmias and atrial septal defect caused by haploinsufficiency of the cardiac transcription factor $\mathrm{Csx} / \mathrm{Nkx} 2.5$. Cold Spring Harb. Symp. Quant. Biol. 67:317-325.

34. Oosthoek, P.W., Viragh, S., Lamers, W.H., and Moorman, A.F. 1993. Immunohistochemical delineation of the conduction system. II: The atrioventricular node and Purkinje fibers. Circ. Res. 73:482-491.

35. Litchenberg, W.H., et al. 2000. The rate and anisotropy of impulse propagation in the postnatal terminal crest are correlated with remodeling of Cx43 gap junction pattern. Cardiovasc. Res. 45:379-387.

36. Bruneau, B.G., et al. 2001. A murine model of HoltOram syndrome defines roles of the T-box transcription factor Tbx5 in cardiogenesis and disease. Cell. 106:709-721.

37. VanderBrink, B.A., et al. 2000. Connexin40-deficient mice exhibit atrioventricular nodal and infraHisian conduction abnormalities. J. Cardiovasc. Electrophysiol. 11:1270-1276.

38. Tamaddon, H.S., et al. 2000. High-resolution optical mapping of the right bundle branch in connexin 40 knockout mice reveals slow conduction in the specialized conduction system. Circ. Res. 87:929-936.

39. Kirchhoff, S., et al. 1998. Reduced cardiac conduction velocity and predisposition to arrhythmias in connexin40-deficient mice. Curr. Biol. 8:299-302. 